\title{
Qualidade de Vida Relacionada à Saúde de Pacientes com Câncer de Mama: Revisão Integrativa da Literatura
}

doi: https://doi.org/10.32635/2176-9745.RBC.2020v66n1.405

\author{
Health-Related Quality of Life of Breast Cancer Patients: Integrative Literature Review \\ Calidad de Vida Relacionada con la Salud de Pacientes con Cáncer de Mama: Revisión Integradora de la Literatura
}

Monique Binotto'; Gilberto Schwartsmann²

Resumo

Introduçáo: $\mathrm{O}$ câncer de mama pode alterar a qualidade de vida relacionada à saúde das pacientes. Objetivo: Compreender o impacto da quimioterapia para câncer de mama na qualidade de vida relacionada à saúde de pacientes. Método: Trata-se de uma revisão integrativa da literatura, compreendendo artigos publicados entre 2007 e 2019, disponíveis nas bases de dados PubMed, LILACS e SciELO. Analisaram-se 25 artigos na íntegra. Resultados: Os questionários mais frequentemente utilizados nos estudos foram o European Organization for Research and Treatment of Cancer Core Quality of Life Questionnaire (EORTC QLQ-C30) e o módulo complementar European Organization for Research and Treatment of Cancer Breast Cancer-specific Quality of Life Questionnaire (EORTC QLQ-BR23). Em relação às alteraçôes da qualidade de vida, a saúde global diminui durante a quimioterapia, mas pode melhorar após o término do tratamento. O aumento dos sintomas é relatado em diversos estudos e prejudicou a qualidade de vida relacionada à saúde das pacientes. Entretanto, os sintomas diminuem após o término da quimioterapia, exceto para algumas escalas. As escalas de imagem corporal, função sexual e funcionamento físico pioram ao longo do tratamento. A qualidade de vida mental/psicológica tem oscilaçôes durante o tratamento, assim como a escala sobre as relaçôes sociais. Conclusáo: A qualidade de vida relacionada à saúde de mulheres com câncer de mama é afetada negativamente pelo tratamento quimioterápico, expressando maior impacto nas escalas de sintomas.

Palavras-chave: Qualidade de Vida; Inquéritos e Questionários; Neoplasias da Mama; Tratamento Farmacológico.

\begin{abstract}
Introduction: Breast cancer can change the health-related quality of life of patients. Objective: To understand the impact of chemotherapy for breast cancer on the health-related quality of life of patients. Method: Integrative review of the literature, comprising articles published between 2007 and 2019, available in PubMed, LILACS and SciELO databases. It were analyzed 25 articles. Results: The questionnaires most frequently used in the studies were the European Organization for Research and Treatment of Cancer Core Quality of Life Questionnaire (EORTC QLQ-C30) and the European Organization for Research and Treatment of Cancer Breast Cancer-specific Quality of Life Questionnaire (EORTC QLQ-BR23) complementary module. In relation to changes in the quality of life, the global health diminishes during chemotherapy, but may improve after the end of the treatment. The increase of the symptoms is reported in several studies and have impaired the patients' health-related quality of life. However, symptoms decrease after chemotherapy ends, except in some scales. Body image, sexual function and physical functioning scales get worse throughout the treatment. Mental/psychological quality of life has oscillations during treatment, as does the scale on social relationships. Conclusion: The health-related quality of life of women with breast cancer is negatively affected by chemotherapy treatment, causing major impact in the scales of symptoms.
\end{abstract}

Key words: Quality of Life; Surveys and Questionnaires; Breast Neoplasms; Drug Therapy.

\section{Resumen}

Introducción: El cáncer de mama puede alterar la calidad de vida relacionada con la salud de las pacientes. Objetivo: Comprender el impacto de la quimioterapia para el cáncer de mama en la calidad de vida relacionado con la salud de los pacientes. Método: Es una revisión integradora de la literatura, que comprende artículos publicados entre 2007 y 2019, disponibles en las bases de datos PubMed, LILACS y SciELO. Se analizaron 25 artículos en su totalidad. Resultados: Los cuestionarios más utilizados fueron European Organization for Research and Treatment of Cancer Core Quality of Life Questionnaire (EORTC QLQ-C30) y European Organization for Research and Treatment of Cancer Breast Cancer-specific Quality of Life Questionnaire (EORTC QLQ-BR23). Con respecto a los cambios en la calidad de vida, la salud general disminuye durante la quimioterapia, pero puede mejorar después del final del tratamiento. El aumento de los síntomas se informa en varios estudios y afecta la calidad de vida relacionado con la salud de los pacientes. Sin embargo, los síntomas disminuyen después de que termina la quimioterapia, excepto en algunas escalas. Las escalas de imagen corporal, la función sexual y la función física empeoran a lo largo del tratamiento. La calidad de vida mental/psicológica tiene oscilaciones durante el tratamiento, al igual que la escala en las relaciones sociales. Conclusión: La calidad de vida relacionada con la salud de las mujeres con cáncer de mama se ve afectada negativamente por el tratamiento quimioterapéutico, que expresa un mayor impacto en las escalas de los síntomas.

Palabras clave: Calidad de Vida; Encuestas y Cuestionarios; Neoplasias de la Mama; Quimioterapia.

${ }^{1}$ Hospital de Clínicas de Porto Alegre (HCPA). Porto Alegre (RS), Brasil. Orcid iD: https://orcid.org/0000-0002-5799-5390

${ }^{2}$ HCPA. Porto Alegre (RS), Brasil. Orcid iD: https://orcid.org/0000-0002-7850-1644

Endereço para correspondência: Monique Binotto. Centro de Pesquisa da Serra Gaúcha. Rua Vinte de Setembro, 2304. Caxias do Sul (RS), Brasil. CEP 95020-450. E-mail: moniquebinotto@gmail.com 


\section{INTRODUÇÃO}

O câncer de mama é o tumor mais incidente e com maior mortalidade em mulheres no mundo todo ${ }^{1,2}$. De etiologia multifatorial, o desenvolvimento do câncer de mama pode envolver fatores biológicos e endócrinos relacionados à vida reprodutiva, ao comportamento e ao estilo de vida. Os fatores de risco mais conhecidos são o envelhecimento, a menarca precoce, a menopausa tardia, a nuliparidade ou a idade avançada na gestação do primeiro filho, o uso prolongado de contraceptivos orais e/ou a reposição hormonal na menopausa, o histórico familiar de câncer de mama e a alta densidade do tecido mamário ${ }^{2,3}$. Além disso, existem genes que demonstraram ser responsáveis pela natureza hereditária de certos cânceres de mama, como o BRCA1 e BRCA2, além dos ATM, PALB2, BRIP1, CHEK, BARD1, que, embora sejam genes menos frequentes, também podem ser responsáveis pelo aumento do risco de câncer de mama ${ }^{4}$. Finalmente, o sedentarismo, o consumo de álcool em excesso e a exposição à radiação ionizante também são considerados agentes potenciais para o desenvolvimento desse câncer ${ }^{2,5}$.

As terapias sistêmicas adjuvantes são eficazes na reduçấo do risco de recorrência do câncer de mama, incluindo a terapia endócrina, a terapia anti-HER2 e a quimioterapia $^{6}$. Já a quimioterapia neoadjuvante para câncer de mama inicial pode tornar a setorectomia mais factível, com menor comprometimento físico e agravos psicológicos na paciente, além dos possíveis benefícios clínicos. Uma recente metanálise comparou os desfechos em longo prazo entre os tratamentos adjuvantes e neoadjuvantes e concluiu que as taxas de mortalidade do câncer de mama são equivalentes ${ }^{7}$.

Entretanto, o tratamento do câncer de mama ocasiona alguns efeitos negativos na recuperaçấo e na qualidade de vida relacionada à saúde (QVRS) das sobreviventes ${ }^{8}$. Independentemente da intenção curativa das terapias, é essencial analisar questôes sobre os efeitos relacionados à toxicidade em longo prazo, que podem afetar a qualidade geral da sobrevida do paciente 9 . Dessa forma, costuma-se afirmar que a quimioterapia é o tipo de tratamento que mais impacta negativamente na QVRS das pacientes com câncer de mama ${ }^{10}$.

Os desfechos reportados pelas pacientes são cada vez mais inclusos nos estudos clínicos, tornando-se importantes na literatura científica. Esse aumento da inclusão da análise de QVRS, em estudos clínicos, pode ser evidenciado pelo acréscimo significativo desse tema durante o American Society of Clinical Oncology (ASCO) Annual Meeting. Em uma pesquisa realizada na plataforma eletrônica que disponibiliza os resumos apresentados durante o congresso ${ }^{11}$, encontrou-se um aumento do número dos que abordam o tema em questão, entre os anos de 2014 e 2018. Dessa maneira, o número de resumos publicados segundo o termo health related quality of life (QVRS) aumentou de 89 resumos em 2014 para 180 em 2018. Já o número de resumos publicados segundo o termo quality of life; ou seja, qualidade de vida (QV) (que é o termo mais abrangente relacionado ao mesmo assunto) aumentou de 322 resumos em 2014 para 410 resumos no ano de 2018.

Sendo assim, o objetivo deste estudo é revisar a literatura a fim de avaliar o impacto do tratamento quimioterápico na QVRS de pacientes com câncer de mama. Além disso, visou-se a identificar quais são os instrumentos mais frequentemente utilizados para mensurar essas alteraçóes.

\section{MÉTODO}

O método utilizado foi a revisão integrativa da literatura. A questáo norteadora foi: "Qual o impacto do tratamento quimioterápico na QVRS de pacientes com câncer de mama?” Para pesquisar os artigos a serem revisados, foram utilizadas as bases de dados PubMed, LILACS e SciELO. Identificaram-se os artigos publicados durante o período de 2007 a 2019, por meio dos descritores qualidade de vida, neoplasias da mama e quimioterapia (tratamento farmacológico, quimioterapia farmacológica, quimioterapia combinada ou protocolos de quimioterapia combinada antineoplásica) e os descritores correspondentes em língua inglesa (Quality of Life, Breast Neoplasms, Drug Therapy, Chemotherapy Adjuvant, Chemotherapy Cancer, Regional Perfusion, Antineoplastic Combined Chemotherapy Protocols, Antineoplastic Agents, Drug Therapy Combination).

Foram considerados critérios de elegibilidade para seleção dos artigos: 1) que as pacientes fossem mulheres; 2) que fossem portadoras de câncer de mama (independente do estadiamento clínico); 3) que os estudos abordassem a quimioterapia para tratamento do câncer de mama; 4) que os estudos tenham realizado uma avaliaçáo comparativa da QVRS em dois ou mais momentos do tratamento quimioterápico; 5) que a avaliação do impacto da quimioterapia fosse a temática principal do estudo. Foram excluídos da análise artigos de opinião, editoriais, relatos de casos, cartas ao editor e comentários. A descrição completa dos artigos incluídos e excluídos está disposta na Figura 1.

\section{RESULTADOS}

Oito diferentes questionários para avaliação da QVRS foram utilizados nos estudos incluídos nesta revisão. 


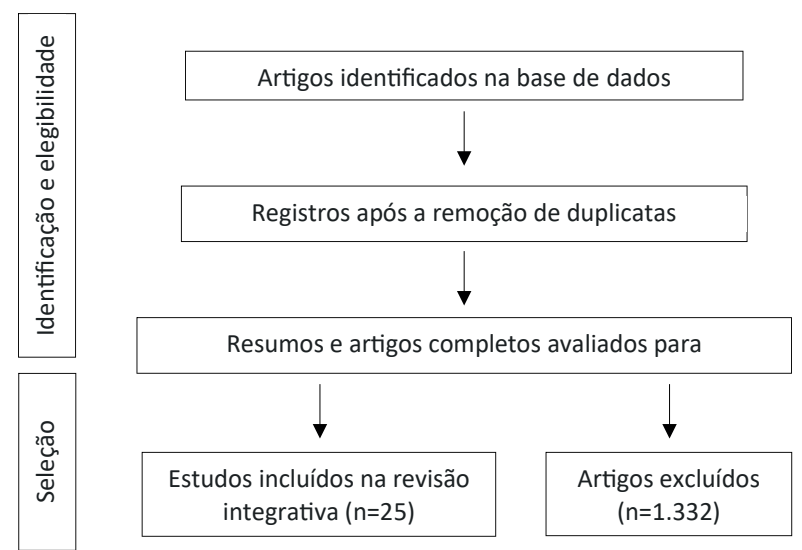

Figura 1. Fluxograma de seleção dos artigos da revisão integrativa da literatura

Quatorze estudos utilizaram o questionário European Organization for Research and Treatment of Cancer Core Quality of Life Questionnaire (EORTC QLQ-C30) ${ }^{12-25}$, mas somente nove estudos utilizaram o módulo complementar para câncer de mama European Organization for Research and Treatment of Cancer Breast Cancer-specific Quality of Life Questionnaire (EORTC QLQ-BR23) ${ }^{12,17,18,20-25}$. Seis estudos utilizaram o questionário Functional Assessment of Cancer Therapy - Breast (FACT-B ${ }^{19,25-29}$ e dois, o Functional Assessment of Cancer Therapy - General (FACT-G) $)^{30,31}$. Já o questionário Medical Outcomes Study 36-item Short Form 36 (SF-36) foi utilizado por três estudos ${ }^{32-34}$, assim como o questionário European Quality of Life 5 Dimensions (EQ-5D) ${ }^{16,28,35}$. Ainda, um estudo utilizou o questionário Quality of Life Questionnaire for Cancer Patients Treated with Anti-Cancer Drugs (QOL-ACD) $)^{35}$ e outro, o International Breast Cancer Study Group Quality of Life Core Questionnaire (IBCSG QL core) $^{36}$. O delineamento do estudo mais frequente foi o longitudinal $(\mathrm{n}=10)$, seguido pelos estudos de coorte aninhado em ensaio clínico randomizado (ECR) $(\mathrm{n}=6)$. A síntese metodológica dos artigos está resumida na Tabela 1 .

Tabela 1. Caracterização dos estudos analisados durante a revisão integrativa

\begin{tabular}{|c|c|c|c|c|c|c|}
\hline Autor & N & Delineamento & Objetivo & Questionários & $\begin{array}{l}\text { Método de aplicação dos } \\
\text { questionários }\end{array}$ & Quimioterapia \\
\hline Au et al. ${ }^{17}$ & 3.222 & ECR & $\begin{array}{l}\text { Descrever e comparar } \\
\text { a QVRS em pacientes } \\
\text { com câncer de mama em } \\
\text { estágio inicial }\end{array}$ & $\begin{array}{l}\text { EORTC QLQ-C30 e } \\
\text { EORTC QLQ-BR23 }\end{array}$ & $\begin{array}{l}\text { Linha de base, no início } \\
\text { do } 4^{0} \text { ciclo, ao final da } \\
\text { quimioterapia e } 6,12 \text { e } 24 \\
\text { meses após a quimioterapia }\end{array}$ & Adjuvante \\
\hline $\begin{array}{l}\text { Baena-Cañada } \\
\text { et al. }{ }^{12}\end{array}$ & 50 & $\begin{array}{l}\text { Coorte aninhado em } \\
\text { ECR }\end{array}$ & $\begin{array}{l}\text { Avaliar o efeito da } \\
\text { quimioterapia na QVRS } \\
\text { e em variáveis clínicas }\end{array}$ & $\begin{array}{l}\text { EORTC QLQ-C30 e } \\
\text { EORTC QLQ-BR23 }\end{array}$ & $\begin{array}{l}\text { Antes da quimioterapia, no } \\
\text { ponto médio dos ciclos de } \\
\text { tratamento e ao final do } \\
\text { tratamento }\end{array}$ & Adjuvante \\
\hline $\begin{array}{l}\text { Bastani e } \\
\text { Kiadaliri' }^{13}\end{array}$ & 100 & ECR & $\begin{array}{l}\text { Comparar as diferenças } \\
\text { entre o nível de } \\
\text { QVRS após receber } \\
\text { dois protocolos de } \\
\text { quimioterapia }\end{array}$ & EORTC QLQ-C30 & $\begin{array}{l}\text { Após o término da } \\
\text { quimioterapia e } 4 \text { meses } \\
\text { depois do final do } \\
\text { tratamento quimioterápico }\end{array}$ & Adjuvante \\
\hline Berger et al. ${ }^{33}$ & 196 & $\begin{array}{l}\text { Coorte aninhado em } \\
\text { ECR }\end{array}$ & $\begin{array}{l}\text { Examinar as relações } \\
\text { entre fadiga e QV } \\
\text { física e mental em } \\
\text { diferentes protocolos de } \\
\text { quimioterapia }\end{array}$ & $\begin{array}{l}\text { Piper Fatigue Scale e } \\
\text { SF-36 }\end{array}$ & $\begin{array}{l}48 \text { horas antes do tratamento } \\
\text { e } 4,8 \text { e } 30 \text { dias após } 0 \\
\text { tratamento final }\end{array}$ & Adjuvante \\
\hline Bernhard et al. ${ }^{36}$ & 243 & ECR & $\begin{array}{l}\text { Avaliar a QVRS, } \\
\text { toxicidade e tempo sem } \\
\text { sintomas até o óbito }\end{array}$ & IBCSG QL core & $\begin{array}{l}\text { Antes do início do } \\
\text { tratamento, e aos 3, 6, 9, } 12 \\
\text { e } 18 \text { meses }\end{array}$ & Adjuvante \\
\hline Browall et al. ${ }^{18}$ & 150 & Longitudinal & $\begin{array}{l}\text { Descrever as alterações } \\
\text { da QVRS durante } 0 \\
\text { tratamento e identificar } \\
\text { os melhores preditores } \\
\text { de QV global após o } \\
\text { tratamento }\end{array}$ & $\begin{array}{l}\text { EORTC QLQ-C30, } \\
\text { EORTC QLQ-BR23 e } \\
\text { HADS }\end{array}$ & $\begin{array}{l}\text { Antes do início do } \\
\text { tratamento, durante e após } \\
\text { completarem o tratamento }\end{array}$ & Adjuvante \\
\hline Cámara et al..$^{14}$ & 3.691 & $\begin{array}{l}\text { Coorte aninhado em } \\
\text { ECR }\end{array}$ & $\begin{array}{l}\text { Comparar as curvas de } \\
\text { QV antes, durante e até } \\
\text { um ano após três ciclo } \\
\text { quimioterápicos }\end{array}$ & EORTC QLQ-C30 & $\begin{array}{l}\text { Antes da terapia, após o } 3^{\circ} \\
\text { e } 6^{\circ} \text { ciclo e após } 3,6,9 \text { e } 12 \\
\text { meses }\end{array}$ & Adjuvante \\
\hline
\end{tabular}


Tabela 1. continuação

\begin{tabular}{|c|c|c|c|c|c|c|}
\hline Autor & $\mathbf{N}$ & Delineamento & Objetivo & Questionários & $\begin{array}{c}\text { Método de aplicação dos } \\
\text { questionários }\end{array}$ & Quimioterapia \\
\hline Ganz et al. ${ }^{26}$ & 300 & ECR & $\begin{array}{l}\text { Comparar o efeito dos } \\
\text { tratamentos sobre a QV } \\
\text { e histórico menstrual }\end{array}$ & FACT-B e FACT- TOI & $\begin{array}{l}\text { Antes do início do } \\
\text { tratamento, após } 04^{0} \text { ciclo e } \\
\text { a cada } 6 \text { meses até } 24 \text { meses } \\
\text { de seguimento }\end{array}$ & Adjuvante \\
\hline $\begin{array}{l}\text { Gaton-Johansson } \\
\text { et al. } .^{19}\end{array}$ & 30 & Longitudinal & $\begin{array}{l}\text { Determinar se há uma } \\
\text { diferença na intensidade } \\
\text { dos sintomas e QV } \\
\text { durante a quimioterapia }\end{array}$ & $\begin{array}{l}\text { EORTC QLQ-C30, FACT- } \\
\text { B, HADS, ISI, POM } \\
\text { e VAS }\end{array}$ & $\begin{array}{l}\text { Antes do início do } \\
\text { tratamento, no ponto médio } \\
\text { da quimioterapia e uma } \\
\text { semana após a conclusão da } \\
\text { quimioterapia }\end{array}$ & SI \\
\hline Gozzo et al. ${ }^{20}$ & 79 & Longitudinal & $\begin{array}{l}\text { Avaliar a QVRS e } \\
\text { identificar a ocorrência } \\
\text { de náuseas e vômitos } \\
\text { durante o tratamento }\end{array}$ & $\begin{array}{l}\text { EORTC QLQ-C30 e } \\
\text { EORTC QLQ-BR23 }\end{array}$ & $\begin{array}{l}\text { Antes do início do } \\
\text { tratamento, no ponto médio } \\
\text { e ao final do tratamento }\end{array}$ & $\begin{array}{l}\text { Adjuvante ou } \\
\text { neoadjuvante }\end{array}$ \\
\hline Hagiwara et al. ${ }^{16}$ & 380 & $\begin{array}{l}\text { Coorte aninhado em } \\
\text { ECR }\end{array}$ & $\begin{array}{l}\text { Investigar o impacto } \\
\text { de eventos adversos na } \\
\text { utilidade da saúde e } \\
\text { na QVRS em pacientes } \\
\text { com câncer de mama } \\
\text { metastático submetidas } \\
\text { à quimioterapia de } \\
\text { primeira linha }\end{array}$ & $\begin{array}{l}\text { EORTC QLQ-C30 e } \\
\text { EQ-5D }\end{array}$ & $\begin{array}{l}\text { Na linha de base, } 3,6 \mathrm{e} \\
12 \text { meses após o início do } \\
\text { tratamento }\end{array}$ & Adjuvante \\
\hline Hall et al. ${ }^{21}$ & 830 & ECR & $\begin{array}{l}\text { Explorar o perfil } \\
\text { de toxicidade da } \\
\text { quimioterapia e o } \\
\text { impacto na QVRS }\end{array}$ & $\begin{array}{l}\text { EORTC QLQ-C30, } \\
\text { EORTC QLQ-BR23 e } \\
\text { HADS }\end{array}$ & $\begin{array}{l}\text { Na linha de base, após os } \\
\text { ciclos } 4 \text { e } 8 \text { da quimioterapia, } \\
\text { aos } 9,12,18,24 \text { meses e até } \\
6 \text { anos de seguimento }\end{array}$ & Adjuvante \\
\hline Hatam et al. ${ }^{15}$ & 100 & Longitudinal & $\begin{array}{l}\text { Comparar dois esquemas } \\
\text { de quimioterapia em } \\
\text { pacientes com câncer } \\
\text { de mama e analisar } \\
\text { a toxicidade desses } \\
\text { tratamentos e observar a } \\
\text { QV relacionada à saúde } \\
\text { do paciente }\end{array}$ & EORTC QLQ-C30 & $\begin{array}{l}\text { Durante a primeira sessão } \\
\text { de quimioterapia e após } 4 \\
\text { meses de acompanhamento }\end{array}$ & Adjuvante \\
\hline Ho et al. ${ }^{30}$ & 269 & $\begin{array}{l}\text { Subanálise de estudo } \\
\text { longitudinal }\end{array}$ & $\begin{array}{l}\text { Comparar a saúde } \\
\text { psicológica e a QVRS e } \\
\text { determinar a relação } \\
\text { entre ansiedade, } \\
\text { depressão e QV durante } \\
\text { e após um ano do } \\
\text { término do tratamento }\end{array}$ & FACT-G e HADS & $\begin{array}{l}\text { Durante e após } 1 \text { ano do } \\
\text { tratamento }\end{array}$ & Adjuvante \\
\hline Huang et al. ${ }^{34}$ & 121 & Longitudinal & $\begin{array}{l}\text { Comparar sintomas } \\
\text { e QV entre pacientes } \\
\text { que receberam terapia } \\
\text { alvo, quimioterapia ou } \\
\text { terapia combinada }\end{array}$ & SF-36 & $\begin{array}{l}\text { Antes do tratamento, } 4 \text { e } 12 \\
\text { semanas após o início }\end{array}$ & SI \\
\hline Klemp et al. ${ }^{32}$ & 20 & Longitudinal & $\begin{array}{l}\text { Examinar as alterações } \\
\text { nas funções cognitivas } \\
\text { subjetivas e objetivas e } \\
\text { na QVRS das mulheres } \\
\text { na pré e perimenopausa, } \\
\text { recebendo quimioterapia } \\
\text { para o câncer de mama } \\
\text { e explorar possíveis } \\
\text { preditores de alterações } \\
\text { cognitivas }\end{array}$ & $\begin{array}{l}\text { BCPT, BDI, BFI, CDS, } \\
\text { FACT-COG, HSCS, } \\
\text { MDASI e SF-36 }\end{array}$ & $\begin{array}{l}\text { Antes da quimioterapia, } \\
\text { após o ciclo } 3 \text {, dentro } \\
\text { de } 2-3 \text { semanas após a } \\
\text { quimioterapia adjuvante e } \\
\text { mais de } 8 \text { anos depois }\end{array}$ & $\begin{array}{l}\text { Neoadjuvante e } \\
\text { adjuvante }\end{array}$ \\
\hline
\end{tabular}


Tabela 1. continuação

\begin{tabular}{|c|c|c|c|c|c|c|}
\hline Autor & $\mathbf{N}$ & Delineamento & Objetivo & Questionários & $\begin{array}{c}\text { Método de aplicação dos } \\
\text { questionários }\end{array}$ & Quimioterapia \\
\hline Kornblith et al. ${ }^{22}$ & 350 & Subanálise de ECR & $\begin{array}{l}\text { Subanálise do ECR para } \\
\text { avaliar se o tratamento } \\
\text { com capecitabina } \\
\text { é associado a uma } \\
\text { QVRS melhor do que a } \\
\text { quimioterapia padrão }\end{array}$ & $\begin{array}{l}\text { EORTC QLQ-C30, } \\
\text { EORTC QLQ-BR23 e } \\
\text { HADS }\end{array}$ & $\begin{array}{l}\text { Antes do tratamento, no } \\
\text { ponto médio do tratamento, } \\
\text { depois de } 1 \text { mês pós- } \\
\text {-tratamento, e aos } 12,18 \text { e } \\
24 \text { meses de seguimento }\end{array}$ & Adjuvante \\
\hline Leinert et al. ${ }^{23}$ & 1363 & Subanálise de ECR & $\begin{array}{l}\text { Comparar a QVRS em } \\
\text { pacientes recebendo } \\
\text { quimioterapia adjuvante }\end{array}$ & $\begin{array}{l}\text { EORTC QLQ-C30 e } \\
\text { EORTC QLQ-BR23 }\end{array}$ & $\begin{array}{l}\text { Antes do início do } \\
\text { tratamento, antes do } 4^{0} \text { ciclo } \\
\text { ou } 5^{\circ} \text { ciclo de quimioterapia, } \\
4 \text { semanas após a } \\
\text { quimioterapia e } 6 \text { semanas } \\
\text { após a radioterapia }\end{array}$ & Adjuvante \\
\hline Montazeri et al. ${ }^{24}$ & 167 & Longitudinal & $\begin{array}{l}\text { Examinar o impacto } \\
\text { do diagnóstico e } \\
\text { tratamentos na QVRS }\end{array}$ & $\begin{array}{l}\text { EORTC QLQ-C30 e } \\
\text { EORTC QLQ-BR23 }\end{array}$ & $\begin{array}{l}\text { Após o diagnóstico de câncer } \\
\text { de mama, } 3 \text { meses após } \\
0 \text { início do tratamento e } \\
1 \text { ano após o término do } \\
\text { tratamento (seguimento de } \\
18 \text { meses) }\end{array}$ & Adjuvante \\
\hline Perroud et al. ${ }^{27}$ & 20 & $\begin{array}{l}\text { Coorte aninhado em } \\
\text { ECR }\end{array}$ & $\begin{array}{l}\text { Identificar as mudanças } \\
\text { na QVRS durante a } \\
\text { quimioterapia }\end{array}$ & BPI e FACT-B & $\begin{array}{l}\text { Antes do início do } \\
\text { tratamento, no ponto médio } \\
\text { e ao final do tratamento }\end{array}$ & Adjuvante \\
\hline Sanford et al. ${ }^{31}$ & 80 & Longitudinal & $\begin{array}{l}\text { Observar a qualidade } \\
\text { do sono em pacientes } \\
\text { com câncer de mama em } \\
\text { quimioterapia adjuvante } \\
\text { e avaliar sua relação } \\
\text { com a QVRS }\end{array}$ & $\begin{array}{l}\text { HADS, FACT-ES, FACT-F, } \\
\text { FACT-G e PSQI }\end{array}$ & $\begin{array}{l}\text { 3-14 dias antes do início } \\
\text { da quimioterapia, no } 4^{\circ} \\
\text { ciclo quimioterápico e } 6 \\
\text { meses após o início da } \\
\text { quimioterapia }\end{array}$ & Adjuvante \\
\hline Shiroiwa et al. ${ }^{28}$ & 299 & $\begin{array}{l}\text { Coorte aninhado em } \\
\text { ECR }\end{array}$ & $\begin{array}{l}\text { Analisar a QVRS e } 0 \\
\text { efeito da quimioterapia } \\
\text { adjuvante nas pacientes } \\
\text { de um ECR }\end{array}$ & EQ-5D e FACT-B & $\begin{array}{l}\text { Antes da administração da } \\
\text { quimioterapia nos ciclos } \\
3,5 \text { e } 7 \text {, após } 7 \text { meses e } \\
1 \text { ano após o início da } \\
\text { quimioterapia }\end{array}$ & Adjuvante \\
\hline Tachi et al. ${ }^{35}$ & 48 & Longitudinal & $\begin{array}{l}\text { Esclarecer o impacto } \\
\text { de eventos adversos } \\
\text { associados ao início de } \\
\text { quimioterapia na QVRS }\end{array}$ & EQ-5D e QOL-ACD & $\begin{array}{l}\text { Antes e depois do primeiro } \\
\text { ciclo de quimioterapia }\end{array}$ & $\begin{array}{l}\text { Adjuvante e } \\
\text { neoadjuvante }\end{array}$ \\
\hline Winters et al. ${ }^{25}$ & 182 & Coorte & $\begin{array}{l}\text { Avaliar se há diferenças } \\
\text { na QVRS após a } \\
\text { reconstrução imediata } \\
\text { da mama }\end{array}$ & $\begin{array}{l}\text { BIS, EORTC QLQ-C30 } \\
\text { e EORTC QLQ-BR23, } \\
\text { FACT-B e HADS }\end{array}$ & $\begin{array}{l}\text { Antes da operação cirúrgica } \\
\text { e aos } 3,6 \text { e } 12 \text { meses após } \\
\text { a cirurgia }\end{array}$ & $\begin{array}{l}\text { Adjuvante e } \\
\text { neoadjuvante }\end{array}$ \\
\hline Zhang et al. ${ }^{29}$ & 88 & Longitudinal & $\begin{array}{l}\text { Investigar ansiedade, } \\
\text { depressão e QV durante } \\
\text { os diferentes períodos } \\
\text { intermitentes entre a } \\
\text { quimioterapia }\end{array}$ & FACT-B, SAS e SDS & $\begin{array}{l}\text { Nos } 2^{0}, 3^{0}, 4^{0}, 5^{\circ} \text { e } 6^{0} \text { ciclos } \\
\text { da terapia, sempre ao } \\
\text { primeiro dia do ciclo }\end{array}$ & Adjuvante \\
\hline
\end{tabular}

Legendas: BCPT: Cognitive Problems Scale from the Breast Cancer Prevention Trial; BDI: Beck Depression Inventory I; BFI: Brief Fatigue Inventory; BPI: Brief Pain Inventory; BIS: Body Image Scale; CDS: Cognitive Difficulties Scale; ECR: Ensaio Clínico Randomizado; EORTC: European Organization for Research and Treatment of Cancer; EORTC QLQ-BR23: EORTC Breast Cancer-specific Quality of Life Questionnaire; EORTC QLQ-C30: EORTC Core Quality of Life Questionnaire; EQ-5D: European Quality of Life 5 Dimensions; FACT: Functional Assessment of Cancer Therapy; FACT-B: FACT Breast; FACT-COG: Functional Assessment for Cancer Therapy-Cognition; FACT-ES: FACT Endocrine Subscale; FACT-F: FACT Fatigue; FACT-G: FACT General; HADS: The Hospital Anxiety and Depression Scale; HSCS: The High Sensitivity Cognitive Screen; IBCSG QL core: International Breast Cancer Study Group Quality of Life Core Questionnaire; ISI: The Insomnia Severity Index; MDASI: MDAnderson Symptom Inventory; POM: Pain-O-Meter; PSQI: Pittsburgh Sleep Quality Index; QOL-ACD: Quality of Life Questionnaire for Cancer Patients Treated with Anti-Cancer Drugs; QV: Qualidade de Vida; QVRS: Qualidade de Vida Relacionada à Saúde; SAS: Self-rating Anxiety Scale; SDS: Self-rating Depression Scale; SF-36: Medical Outcomes Study 36-item Short Form; SI: Sem informaçăo; TOI: Trial Outcome Index; VAS: Visual Analogue Scale. 
Em relação às alteraçôes da QVRS, a saúde global diminuiu durante a quimioterapia ${ }^{12,14,15,17-19,21,23,25}$, mas pode melhorar após o término do tratamento ${ }^{14,17,25,36}$. Além disso, a saúde global pode ser impactada negativamente pelos eventos adversos ocasionados pela quimioterapia ${ }^{16}$. $\mathrm{O}$ aumento dos sintomas relacionados à terapia sistêmica é relatado em diversos estudos ${ }^{12,15,17-19,21,23,26,34}$ e prejudicou a QVRS das pacientes. Entre os estudos que avaliaram a QVRS em períodos que compreendiam o pós-tratamento, a maioria demonstrou que os sintomas diminuem após o término da quimioterapia ${ }^{17,19,21,26}$, exceto para algumas escalas, como a depressão, a fadiga e a interferência da dor nas atividades diárias, cujos escores podem aumentar ao término da quimioterapia em comparação com a linha de base $^{19}$. Quanto à escala de imagem corporal, os resultados agravaram-se com o tratamento ${ }^{12,18,24}$. A imagem corporal e a funçáo sexual foram as escalas da QVRS que apresentaram o maior período temporal para a recuperaçáo dos escores aos níveis basais ${ }^{21}$, ou que não demonstraram recuperação durante o período avaliado ${ }^{24}$. Apesar do funcionamento físico, escalas de atividade ou condição física diminuírem durante a quimioterapia ${ }^{12,18,29,30,35}$, foi relatada a melhora nessa escala após o término do tratamento em alguns estudos ${ }^{23,33,37}$. Entretanto, a piora pós-tratamento foi descrita em um dos estudos ${ }^{19}$.

A QV mental, condição ou funcionamento psicológico, funçáo emocional ou cognitiva (a nomenclatura difere dependendo do questionário utilizado) também indicaram alteraçóes em decorrência do tratamento. Durante o tratamento, identificou-se a reduçáo da QV mental, que apresentou melhora após o término da quimioterapia ${ }^{32,33}$. Outros autores relataram que a condição psicológical emocional diminuiu após o tratamento ${ }^{24,27,29,31,35}$ ou que a função emocional e o funcionamento cognitivo aumentam somente após determinado período, ao término do tratamento ${ }^{13}$.

Quadro 1. Resumo dos principais resultados dos estudos analisados na revisão integrativa

\begin{tabular}{|c|c|}
\hline $\begin{array}{l}\text { Autor e } \\
\text { referência }\end{array}$ & Resultados \\
\hline Au et al. ${ }^{17}$ & $\begin{array}{l}\text { O funcionamento físico foi um pouco pior para aqueles que receberam ACT, comparado ao } \\
\text { grupo que recebeu } \mathrm{TCH}(\mathrm{p}<0,002) \text {. No ponto médio, o grupo TCH foi pior do que o grupo } \\
\text { ACTH ( } p=0,005) \text {, mas semelhante entre os braços, com recuperação aos } 12 \text { meses. Os escores } \\
\text { de alteração do efeito colateral sistêmico foram melhores nos pacientes tratados com } \mathrm{TCH} \text { em } \\
\text { comparação com ACTH e ACT ao final do tratamento }(p=0,001) \text {, sugerindo maior tolerabilidade. } \\
\text { Todos os braços de tratamento se recuperaram quanto ao efeito colateral sistêmico, funcionamento } \\
\text { físico e na escala global de saúde em } 1 \text { ano e os escores médios de mudança de perspectiva } \\
\text { futura continuaram melhorando ao longo do tratamento e no seguimento }\end{array}$ \\
\hline $\begin{array}{l}\text { Baena- } \\
\text { Cañada et } \\
\text { al. }{ }^{12}\end{array}$ & $\begin{array}{l}\text { O estado de saúde global/QV piorou ao longo do tempo }(p=0,01) \text {, enquanto os escores de } \\
\text { funcionamento físico }(p=0,0001) \text { e imagem corporal }(p=0,002) \text { obtiveram maior impacto } \\
\text { negativo com a quimioterapia. Houve o aumento dos sintomas do tratamento sistêmico } \\
\text { ( } p=0,001) \text {. Alguns deles apresentaram maior impacto negativo e temporal da quimioterapia: } \\
\text { como fadiga }(p=0,004) \text {, falta de apetite }(p=0,025) \text {, náuseas e vômitos }(p=0,05)\end{array}$ \\
\hline $\begin{array}{l}\text { Bastani e } \\
\text { Kiadaliri }^{13}\end{array}$ & $\begin{array}{l}\text { Ao final da quimioterapia, os escores de QVRS melhoraram em todos os cinco aspectos do } \\
\text { estado da função (física, funcional, emocional, cognitiva e social) e estado de saúde global/QV } \\
\text { nos dois grupos. Nos dois braços, a maioria dos progressos foi observada no funcionamento } \\
\text { físico (aumento de } 13 \% \text { em FAC e } 29 \% \text { em TAC). No final da quimioterapia, o escore médio de } \\
\text { QVRS no grupo FAC foi superior a TAC }(p<0,005)\end{array}$ \\
\hline $\begin{array}{l}\text { Berger et } \\
\text { al. }^{33}\end{array}$ & $\begin{array}{l}\text { Baixos escores de } Q V \text { física no início do tratamento (em comparação com o controle - população } \\
\text { geral) e entre o } 4^{\circ} \text { e } 8^{\circ} \text { ciclo, com melhora em } 30 \text { dias após o final da quimioterapia. Houve piora } \\
\text { da } Q V \text { física ao longo do tempo, independentemente do protocolo quimioterápico }(p<0,001) \text {. A } \\
Q V \text { mental das pacientes foi inicialmente semelhante ao controle, com declínio após o } 4^{\circ} \text { ciclo, } \\
\text { que só se recuperou no } 8^{\circ} \text { ciclo e na pós-terapia. A } Q V \text { mental foi impactada ao longo do tempo, } \\
\text { independentemente do protocolo quimioterápico }(p<0,001)\end{array}$ \\
\hline $\begin{array}{l}\text { Bernhard et } \\
\text { al. }^{36}\end{array}$ & $\begin{array}{l}\text { Não houve diferenças basais entre os tratamentos. Houve redução da QVRS durante o tratamento, } \\
\text { com melhora acentuada } 3 \text { meses após a quimioterapia (em ambos os grupos). Essa recuperação } \\
\text { foi maior e mais rápida na coorte DI-EC, em comparação com a coorte SD-CT. A alteração nas } \\
\text { pontuações de enfrentamento após a conclusão da terapia no braço DI-EC melhorou do mês } 3 \\
\text { para o } 6(p<0,01) \text {, enquanto, no braço SD-CT, o escore de enfrentamento melhorou do mês } 6 \\
\text { até o mês } 9(p<0.01) \text {. Os pacientes da coorte DI-CE obtiveram melhores estimativas de saúde } \\
\text { durante todo o acompanhamento }\end{array}$ \\
\hline
\end{tabular}


Quadro 1. continuação

\begin{tabular}{|c|c|}
\hline $\begin{array}{l}\text { Autor e } \\
\text { referência }\end{array}$ & Resultados \\
\hline $\begin{array}{l}\text { Browall et } \\
\text { al. }{ }^{18}\end{array}$ & $\begin{array}{l}\text { Diminuição dos escores de saúde global/QV, funcionamentos (físico, social e cognitivo), } \\
\text { desempenho funcional, imagem corporal e ansiedade, além de aumento dos escores de fadiga, } \\
\text { náuseas e vômitos, dispneia, perda de apetite, constipação, dor, efeitos sistêmicos, perda de } \\
\text { cabelo e depressão com a quimioterapia (em comparação da linha de base, } 3^{\circ} \text { e } 6^{\circ} \text { ciclos). Na } \\
\text { linha base, algumas escalas foram correlacionadas com melhor saúde geral/QV ao final do } \\
\text { tratamento: desempenho funcional }(p<0,05) \text { e os funcionamentos físico ( }<0,01) \text {, emocional } \\
\text { ( } p<0,05) \text { e social ( } p<0,05) \text {; ou seja, quanto melhores estivessem na linha base, melhor a saúde/ } \\
\text { geral QV ao final da quimioterapia. Relação inversamente proporcional para fadiga }(p<0,01) \text {, } \\
\text { depressão }(p<0,05) \text { e ansiedade }(p<0,05)\end{array}$ \\
\hline $\begin{array}{l}\text { Cámara et } \\
\text { al. }^{14}\end{array}$ & $\begin{array}{l}\text { Em todos os momentos, a QV global média foi maior com o FEC-DG do que com o FEC-D } \\
(p=0,05) \text { e, no } 3^{\circ} \text { seguimento, essa diferença atingiu seu máximo de dois pontos }(p=0,02) \text {, mas } \\
\text { ambos os resultados são abaixo do limiar de relevância clínica. Mulheres com FEC-DG relataram } \\
\text { menos dor e fadiga e uma função física melhor no } 3^{\circ} \text { seguimento }(p=0,001)\end{array}$ \\
\hline Ganz et al. ${ }^{26}$ & $\begin{array}{l}\text { O tempo ( } p<0,001) \text {, protocolo quimioterápico }(p<0,001) \text { e combinação cirurgia/radiação }(p<0,024) \\
\text { foram preditores da mudança da linha de base até os } 24 \text { meses de seguimento da } Q V R S \text {. Os pacientes } \\
\text { do grupo ACT reduziram o escore de QVRS em relação à linha de base (tamanho do efeito } 0,2 \text { ), assim } \\
\text { como os pacientes em AT (tamanho do efeito } 0,1) \text {. A interação tempo por tratamento demonstrou } \\
\text { que o bem-estar físico e funcional dos pacientes com TAC e AT retornou à linha de base aos } 6 \text { meses, } \\
\text { enquanto o dos pacientes com ACT retornou à linha de base aos } 12 \text { meses }(p<0,001) \text {. Os sintomas } \\
\text { aumentaram ao longo dos tratamentos, sem retorno aos níveis basais }\end{array}$ \\
\hline $\begin{array}{l}\text { Gaton- } \\
\text { - Johansson } \\
\text { et al. }{ }^{19}\end{array}$ & $\begin{array}{l}\text { Agravo da interferência da dor nas atividades diárias }(p=0,001) \text {, além de aumento da fadiga } \\
\text { ( } p<0,001) \text {, náuseas presentes }(p=0,005) \text {, histórico de náuseas }(p=0,006) \text { e insônia }(p=0,024) \\
\text { com a quimioterapia. Houve maior intensidade de dor, náuseas, histórico de fadiga e insônia } \\
\text { no ponto médio da quimioterapia. Mais depressão, fadiga e interferência da dor nas atividades } \\
\text { diárias na pós-terapia. Piora da saúde global/QV ( } p=0,001) \text {, bem-estar físico }(p<0,001) \text {, } \\
\text { funcional ( } p<0,001) \text { e familiar/social }(p=0,001) \text { ao longo do tratamento }\end{array}$ \\
\hline $\begin{array}{l}\text { Gozzo et } \\
\text { al. }{ }^{20}\end{array}$ & $\begin{array}{l}\text { De modo geral, a QVRS ficou estável durante o tratamento. Exceção para a maior presença de } \\
\text { náuseas e vômitos ao menos uma vez, durante o tratamento: EC-T/EC-TH (início/meio -9,3746, } \\
p=0,0118 \text { e início/final }-9,1052, p=0,0151 \text { ) e FEC (início/meio }-18,2789, p=0,0001 \text { e início/ } \\
\text { final }-12,9026, p=0,0055 \text { ) }\end{array}$ \\
\hline $\begin{array}{l}\text { Hagiwara et } \\
\text { al. }{ }^{16}\end{array}$ & 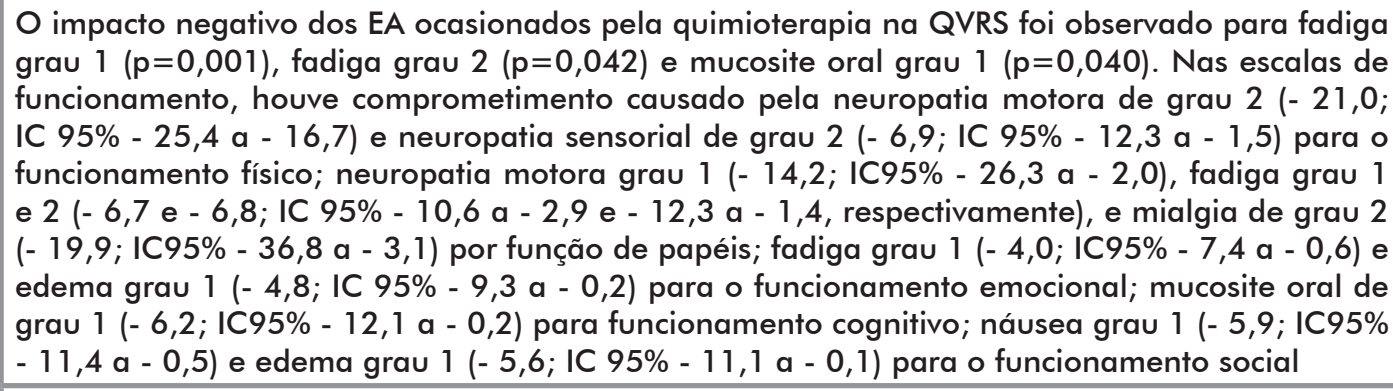 \\
\hline Hall et al. ${ }^{21}$ & $\begin{array}{l}\text { Comprometimento da saúde global/QV entre a linha de base e o tratamento: pior para o grupo } \\
\text { FEC-D em comparação com os controles no ciclo } 8(p<0,0001) \text {. A recuperação aos níveis basais } \\
\text { ocorreu nos dois protocolos por } 12 \text { meses, com escores inalterados em relação aos valores basais } \\
\text { aos } 2 \text { e } 6 \text { anos. Aumento dos efeitos colaterais sistêmicos em FEC-D desde o início até o meio } \\
\text { do tratamento. No ciclo } 8 \text {, esse aumento também foi significantemente maior nos pacientes com } \\
\text { FEC-D ( } p=0,005) \text {. Todos os efeitos sistêmicos, exceto a xerostomia, diminuíram no pós-tratamento, } \\
\text { mas sem retornarem ao nível basal. A imagem corporal e o funcionamento sexual demandaram } \\
\text { maior tempo de resolução. Nenhuma alteração adicional foi identificada aos } 6 \text { anos }\end{array}$ \\
\hline $\begin{array}{l}\text { Hatam et } \\
\text { al. }{ }^{15}\end{array}$ & $\begin{array}{l}\text { Após o acompanhamento dos pacientes por } 4 \text { meses, os resultados indicaram que, apesar de ter o } \\
\text { mesmo escore médio de QVRS no início da quimioterapia, a QVRS no grupo TAC foi pior, com maior } \\
\text { toxicidade. A QVRS após o } 1^{a} \text { ciclo de quimioterapia não apresentou diferenças entre TAC ou FAC, } \\
\text { com escore médio semelhante. Ao final da quimioterapia }\left(6^{\circ} \text { ciclo), a QVRS em ambos os grupos se }\right. \\
\text { deteriorou como resultado dos efeitos colaterais. No entanto, a diminuição foi maior no grupo TAC, } \\
\text { em todos os aspectos do estado funcional do paciente: função física }(p=0,002) \text {, funcional }(p=0,006) \text {, } \\
\text { emocional }(p=0,007) \text {, cognitiva }(p=0,02) \text {, social }(p=0,002) \text { e estado de saúde global/QV }(p<0,001)\end{array}$ \\
\hline
\end{tabular}


Quadro 1. continuação

\begin{tabular}{|c|c|}
\hline $\begin{array}{l}\text { Autor e } \\
\text { referência }\end{array}$ & Resultados \\
\hline Ho et al. ${ }^{30}$ & $\begin{array}{l}\text { Níveis mais baixos de QVRS durante a quimioterapia. Maior ansiedade foi associada ao menor } \\
\text { bem-estar físico (durante o tratamento: beta }-0,25 p<0,001 \text {, após: beta } 0,21 p=0,03 \text { ), emocional } \\
\text { (durante: beta }-0,57 p<0,001 \text {, após beta }-0,72 p<0,001 \text { ) e funcional (durante: beta }-0,21 p=0,001 \\
\text { e após: beta }-0,32 p<0,001 \text { ). Maiores níveis de depressão foram associados ao menor bem-estar } \\
\text { físico (durante: beta }-0,34, p<0,001 \text {, após; beta }-0,37 p<0,001 \text { ), social/familiar (durante: beta } \\
-0.26 p=0.001 \text {, após: beta }-0,34 p=0,001 \text { ), emocional (somente durante o tratamento: beta }-0,22 \\
p<0,001 \text { ) e funcional (durante: beta }-0,48 p<0,001 \text {, após: beta }-0,43 p<0,001 \text { ) }\end{array}$ \\
\hline $\begin{array}{l}\text { Huang et } \\
\text { al. }{ }^{34}\end{array}$ & $\begin{array}{l}\text { A gravidade dos sintomas aumentou ao longo do tempo entre os pacientes que receberam } \\
\text { quimioterapia (média: } 26,2 \pm 15,5 \text { para pré-tratamento, } 31,1 \pm 18,4 \text { nas } 4 \text { semanas após o início } \\
\text { do tratamento e } 34,2 \pm 22,2 \text { nas } 12 \text { semanas após o início do tratamento; } p=0,02 \text { ) }\end{array}$ \\
\hline $\begin{array}{l}\text { Klemp et } \\
\text { al. }{ }^{32}\end{array}$ & $\begin{array}{l}\text { Maior QV foi correlacionada com melhor função cognitiva subjetiva }(r=0,705, p=0,002) \text { e } \\
\text { menor índice de massa corporal }(r=-0,502, p=0,017) \text {. Os escores do componente físico da } \\
\text { QVRS melhoraram ao longo do tempo }(p=0,037) \text {. Não foi demonstrada alteração no escore do } \\
\text { componente mental }\end{array}$ \\
\hline $\begin{array}{l}\text { Kornblith et } \\
\text { al. }{ }^{22}\end{array}$ & $\begin{array}{l}\text { Os pacientes tratados com capecitabina obtiveram melhor estado de saúde global/QV no meio } \\
\text { ( } p<0,001) \text { e ao final do tratamento }(p<0,001) \text {, além de menos efeitos adversos relacionados } \\
\text { ao tratamento sistêmico no ponto médio }(p<0,001) \text { e ao final }(p<0,001) \text {. Ainda, o grupo } \\
\text { de capecitabina apresentou melhor escala funcional }(p \leq 0,002) \text { e social }(p<0,001) \text {, além de } \\
\text { menos fadiga }(p<0,001) \text {, menos náusea e vômito }(p<0,001) \text {, menos constipação }(p \leq 0,004) \text { e } \\
\text { melhor apetite }(p=0,005) \text { do que os pacientes tratados com quimioterapia padrão, no meio do } \\
\text { tratamento e/ou final do tratamento }\end{array}$ \\
\hline $\begin{array}{l}\text { Leinert et } \\
\text { al. }{ }^{23}\end{array}$ & 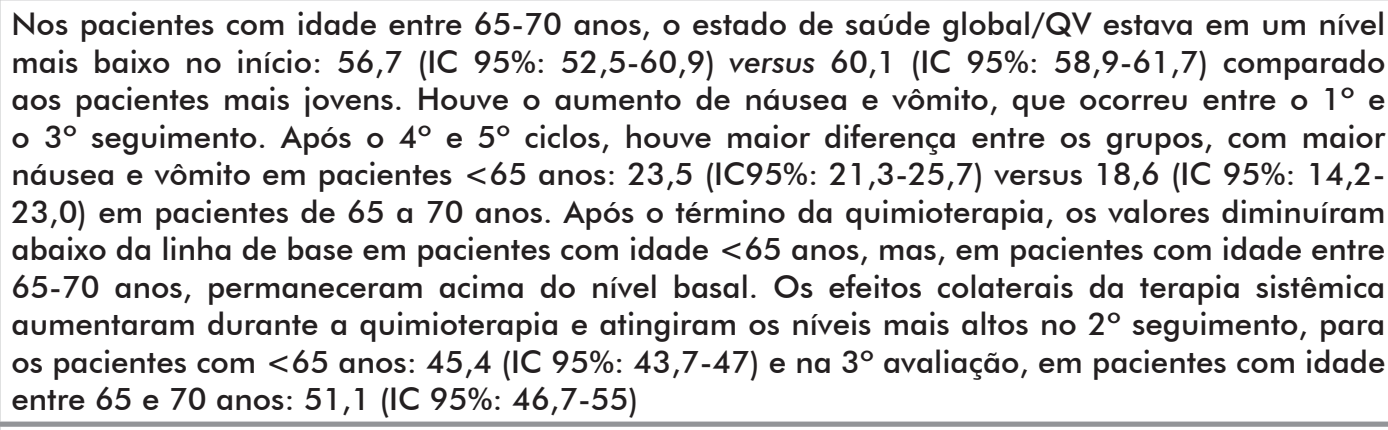 \\
\hline $\begin{array}{l}\text { Montazeri et } \\
\text { al. }^{24}\end{array}$ & $\begin{array}{l}\text { O funcionamento físico melhorou com o tempo }(p=0,001) \text {, enquanto houve deterioração nas } \\
\text { escalas funcionais: emocional }(p=0,001) \text { e cognitiva }(p=0,001) \text {. Os escores globais de } Q V \\
\text { mostraram um quadro flutuante: (início } 59,2 \pm 31,8, T 371,3 \pm 25,6 T 1832,0 \pm 30,2, p=0,001) \text {. } \\
\text { Todos os sintomas aumentaram após } 3 \text { meses de seguimento (náuseas e vômitos, perda de } \\
\text { apetite, efeitos colaterais da terapia sistêmica com } p<0,001 \text { e diarreia } p=0,003 \text { ) ou após } \\
18 \text { meses (fadiga, dor, dispneia, dificuldades no sono, constipação, dificuldades financeiras, } \\
\text { sintomas no braço: } p<0,001 \text { e queda de cabelo: } p=0,003) \text {. A imagem corporal piorou ao longo } \\
\text { do tempo ( }<<0,001) \text {, assim como o funcionamento sexual }(p<0,001) \text { e o aproveitamento sexual } \\
(p<0,001) \text {. A expectativa futura aumentou ao longo do tempo }(p<0,001 \text { ) }\end{array}$ \\
\hline $\begin{array}{l}\text { Perroud et } \\
\text { al. }{ }^{27}\end{array}$ & $\begin{array}{l}\text { Não houve diferença entre o início do tratamento e o ponto médio do tratamento quimioterápico. } \\
\text { Aumento no bem-estar emocional }(p=0,045) \text { e preocupações adicionais entre o ponto médio e } \\
\text { o final da quimioterapia }(p=0,019)\end{array}$ \\
\hline $\begin{array}{l}\text { Sanford et } \\
\text { al. }^{31}\end{array}$ & $\begin{array}{l}\text { Menores escores da QVRS durante o tratamento em comparação com o antes e após o } \\
\text { tratamento ( } p<0,001) \text {. Participantes com má qualidade de sono relataram pior QVRS global, } \\
\text { fadiga e depressão. Das quatro subescalas da QVRS, a função física (subescalas de bem-estar: } \\
p<0,001 \text { ) e funcional }(p<0,001) \text { foram associadas às pontuações globais da qualidade de sono } \\
\text { ao longo do tempo }\end{array}$ \\
\hline $\begin{array}{l}\text { Shiroiwa et } \\
\text { al. }^{28}\end{array}$ & $\begin{array}{l}\text { Os resultados indicaram que os escores de utilidade no grupo DTX foram menores do que em } \\
\text { outros grupos. As pontuações de utilidade nos grupos ACP }(p=0,0048) \text { e ACT }(p=0,0001) \text { foram } \\
\text { mais altas }\end{array}$ \\
\hline Tachi et al. ${ }^{35}$ & $\begin{array}{l}\text { As escalas de atividade }(p=0,003) \text {, condição física }(p<0,001) \text { e psicológica }(p=0,032) \text { da QVRS } \\
\text { diminuíram após a quimioterapia e o escore de relações sociais aumentou }(p<0,001)\end{array}$ \\
\hline
\end{tabular}




\begin{tabular}{|c|c|}
\hline $\begin{array}{l}\text { Autor e } \\
\text { referência }\end{array}$ & Resultados \\
\hline $\begin{array}{l}\text { Winters et } \\
\text { al. }^{25}\end{array}$ & 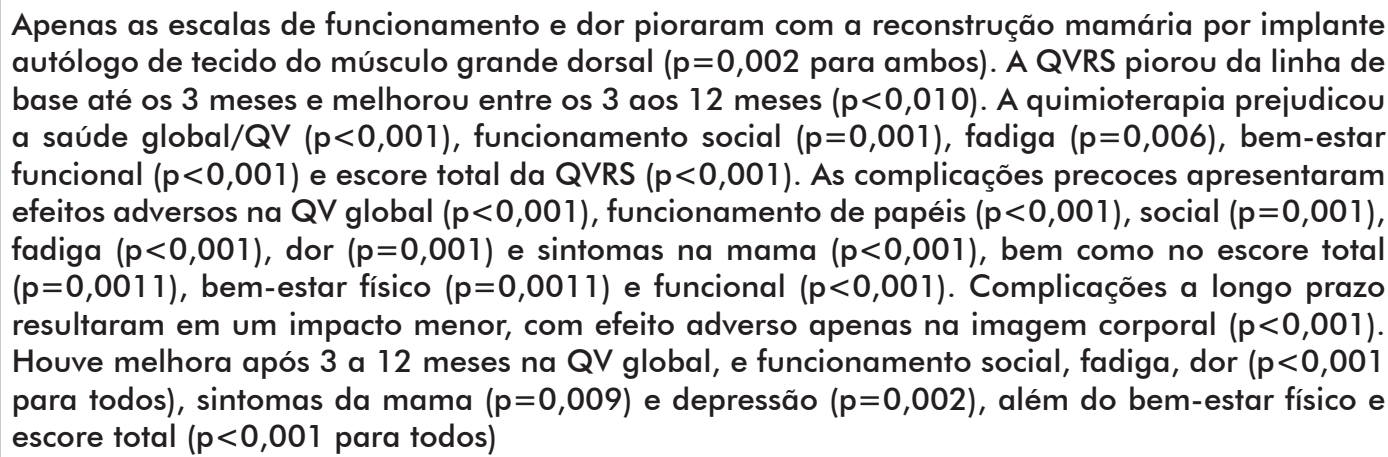 \\
\hline $\begin{array}{l}\text { Zhang et } \\
\text { al. }^{29}\end{array}$ & $\begin{array}{l}\text { Os componentes da QVRS oscilaram durante os seguimentos, terminando com níveis menores } \\
\text { que o basal: bem-estar físico }(p<0,001) \text {, bem-estar social/familiar }(p<0,001) \text {, bem-estar } \\
\text { emocional ( } p<0,001) \text {, bem-estar funcional }(p<0,001) \text { e preocupações adicionais }(p<0,001)\end{array}$ \\
\hline
\end{tabular}

Legendas: ACP: Antraciclina seguida de paclitaxel; ACT: Doxorrubicina e ciclofosfamida seguida por docetaxel; ACTH: Doxorrubicina e ciclofosfamida seguida por docetaxel e trastuzumabe; AT: Doxorrubicina e docetaxel; DI-EC: Epirrubicina adjuvante em dose densa e ciclofosfamida administrada com filgrastim e suporte celular progenitor; DTX: Docetaxel; EA: Eventos adversos; EC-T: Epirrubicina, ciclofosfamida e docetaxel; EC-TH: Epirrubicina, ciclofosfamida, docetaxel e trastuzumabe; FAC: Fluorouracil, doxorrubicina e ciclofosfamida; FEC: Fluorouracil, epirrubicina, ciclofosfamida; FEC-D: Fluorouracil, epirrubicina, ciclofosfamida seguida por docetaxel; FEC-DG: Fluorouracil, epirrubicina, ciclofosfamida seguida por docetaxel e gencitabina; PTX: Paclitaxel; QV: Qualidade de vida; QVRS: Qualidade de vida relacionada à saúde; SD-CT: Quimioterapia baseada em antraciclina; TAC: Docetaxel, doxorrubicina e ciclofosfamida; TCH: Docetaxel mais carboplatina.

Acerca das relaçóes sociais, os resultados diferem dependendo do estudo. Enquanto um estudo relata que a escala aumenta durante o tratamento ${ }^{35}$, outros afirmam que os escores reduzem durante o tratamento, mas aumentam depois do término ${ }^{13,25}$. E, finalmente, há aqueles que acreditam que essa escala piora em decorrência do tratamento ${ }^{18,19,24,29}$. Assim, acredita-se que os impactos na escala relaçóes sociais diferem dependendo do grau de ajustamento psicossocial de cada paciente. O resumo dos resultados de cada artigo está disposto no Quadro 1.

\section{DISCUSSÃO}

Por meio desta revisão da literatura, percebe-se que, na maioria das vezes, o tratamento quimioterápico é responsável pela piora da QVRS de mulheres com câncer de mama. Assim, observou-se que todos os protocolos quimioterápicos afetam uma ou mais de uma escala. Em suma, a saúde global diminui durante a quimioterapia, mas pode melhorar após o término do tratamento. $\mathrm{O}$ aumento dos sintomas é bem relatado e prejudicou a QVRS das pacientes. Entretanto, a maioria dos sintomas diminui após o término da quimioterapia. As escalas de imagem corporal, função sexual e funcionamento físico pioram ao longo do tratamento. A QV mental/psicológica tem oscilaçôes durante o tratamento, assim como a escala sobre as relaçôes sociais. Além disso, a quimioterapia ocasionou efeitos adversos que, apesar de serem transitórios, impactaram na condição física do paciente. Dessa forma, e considerando que os resultados relatados pelos pacientes sobre a QVRS (como o funcionamento físico, emocional e efeitos colaterais relacionados ao tratamento) são cada vez mais importantes, infere-se que as alteraçóes ocasionadas durante a quimioterapia geram mudanças na rotina das pacientes, fato que altera as percepçóes sobre a QVRS.

Um dos fatores que pode impactar de forma negativa a QVRS das pacientes é a questáo emocional, desencadeada após o início do tratamento. Resultado semelhante foi encontrado em uma revisão integrativa da literatura ${ }^{38}$ que relata que os pacientes têm pouco apoio psicossocial, ocasionando uma baixa pontuação no domínio psicológico da QVRS. Além disso, em um estudo ${ }^{31}$ que avaliou escores de depressão e ansiedade, os níveis desses escores pioram durante a quimioterapia, sendo associados com baixos níveis de QVRS. Assim, pode-se inferir que a baixa pontuação na função emocional/cognitiva pode estar associada aos efeitos do tratamento, mas também pode evoluir em função do sofrimento emocional prolongado.

Existem questóes sobre a maneira que a paciente se relaciona com as demais pessoas de seu vínculo social, que podem impactar a QVRS. Ainda que as percepçóes sobre as relaçôes sociais não estejam totalmente esclarecidas, essa escala parece ter relação com o modo com que a paciente enfrenta as etapas do tratamento e com o suporte social proporcionado por seus familiares e amigos. Esse fato está de acordo com os resultados de outra revisáo de literatura ${ }^{39}$. Segundo este estudo, a maneira com que a paciente percebe o contexto familiar é alterada em função do nível de receptividade social da família ${ }^{39}$. Nesse contexto, não há alteraçôes na QVRS caso a paciente 
possua vínculos familiares com baixa receptividade social, mas, caso possua um alto nível de receptividade social, ocorre uma melhora na QVRS (em razão da possibilidade de expressar seus sentimentos, incertezas e a necessidade do apoio familiar).

O presente estudo apresenta como limitação a dificuldade em comparar os resultados dos desfechos entre os estudos, que utilizaram diferentes instrumentos. Ressalta-se que náo há um consenso mundial sobre como a análise de QVRS deve ser mensurada, diversificando o modo como é realizada por cada autor. E que, mesmo os questionários sendo específicos para análise da QVRS, diferentes terminologias são utilizadas na nomenclatura das escalas, além das diferenças entre as questóes utilizadas em cada questionário para calcular a pontuação final dos módulos. Mesmo assim, os instrumentos mais utilizados para avaliar QVRS nos estudos analisados por essa revisão integrativa foram semelhantes aos encontrados em outra revisão ${ }^{40}$.

Com base nos resultados deste estudo, percebe-se que, em relação aos cuidados oncológicos prestados pelos profissionais da saúde aos pacientes, tornou-se imprescindível a elaboração de um plano de cuidados mais abrangente que identifique as alteraçóes emocionais, sociais e de autoimagem, além das limitaçóes físicas e funcionais. Ainda, sugere-se que as questóes relacionadas à doença, como os efeitos colaterais do tratamento, sintomas e o funcionamento sexual devem receber mais atenção, ao considerar a QVRS de pacientes com câncer de mama. Além disso, essa assistência deverá ser estendida a familiares e cônjuges das pacientes, com a abordagem de temáticas educativas, a fim de minimizar o impacto do tratamento nas funçóes sociais e sexuais. Essa reestruturação da assistência prestada exige a formação de equipes multiprofissionais, que possam auxiliar a paciente em todos os aspectos relativos à QVRS, compreendendo a paciente e suas percepçôes de saúde de modo global.

\section{CONCLUSÃO}

A partir desta revisão, conclui-se que a QVRS de mulheres com câncer de mama é afetada negativamente pelo tratamento quimioterápico. Percebe-se que as escalas de sintomas apresentaram as maiores alteraçóes, ao se comparar o início com o final do tratamento. Assim, os dados acerca da avaliação da QVRS fornecem evidências de que algumas decisóes clínicas devem considerar a percepção do paciente sobre a própria saúde.

\section{CONTRIBUIÇÕES}

Ambos os autores contribuíram substancialmente na concepção e no planejamento do estudo, na obtenção, análise e/ ou interpretação dos dados, assim como na redação e/ou revisão crítica e aprovaram a versão final a ser publicada.

\section{DECLARAÇÃO DE CONFLITO DE INTERESSES}

\author{
Nada a declarar. \\ FONTES DE FINANCIAMENTO
}

Não há.

\section{REFERÊNCIAS}

1. Goss PE, Lee BL, Badovinac-Crnjevic T, et al. Planning cancer control in Latin America and the Caribbean. Lancet Oncol. 2013;14(5):391-436. doi: https://doi. org/10.1016/S1470-2045(13)70048-2

2. Instituto Nacional de Câncer José Alencar Gomes da Silva. Estimativa 2020: incidência de câncer no Brasil [Internet]. Rio de Janeiro: INCA; 2019. [acesso 2020 mar. 07]. Disponível em: https://www.inca.gov.br/sites/ ufu.sti.inca.local/files//media/document//estimativa2020-incidencia-de-cancer-no-brasil.pdf

3. Dall GV, Britt KL. Estrogen effects on the mammary gland in early and late life and breast cancer risk. Front Oncol. 2017;7:110. doi: https://doi.org/10.3389/ fonc. 2017.00110

4. Jian W, Shao K, Qin Q, et al. Clinical and genetic characterization of hereditary breast cancer in a Chinese population. Hered Cancer Clin Pract. 2017;15:19. doi: https://doi.org/10.1186/s13053-017-0079-4

5. Picon-Ruiz M, Morata-Tarifa C, Valle-Goffin JJ, et al. Obesity and adverse breast cancer risk and outcome: Mechanistic insights and strategies for intervention. CA Cancer J Clin. 2017;67(5):378-97. doi: https://doi. org/10.3322/caac. 21405

6. Anampa J, Makower D, Sparano JA. Progress in adjuvant chemotherapy for breast cancer: an overview. BMC Med. 2015;13:195. doi: https://doi.org/10.1186/s12916-0150439-8

7. Asselain B, Barlow W, Bartlett J, et al. Long-term outcomes for neoadjuvant versus adjuvant chemotherapy in early breast cancer: meta-analysis of individual patient data from ten randomised trials. Lancet Oncol. 2018;19(1):27-39. doi: http://dx.doi.org/10.1016/ S1470-2045(17)30777-5

8. Chopra I, Kamal KM. A systematic review of quality of life instruments in long-term breast cancer survivors. Health Qual Life Outcomes. 2012;10:14. doi: http:// dx.doi.org/10.1186/1477-7525-10-14

9. Azim HA Jr, Azambuja E, Colozza M, et al. Long-term toxic effects of adjuvant chemotherapy in breast cancer. Ann Oncol. 2011;22(9):1939-47. doi: https://doi. org/10.1093/annonc/mdq683 
10. Muñoz M. Quality of life during treatment in young women with breast cancer. Breast Cancer Res Treat. 2010;123(Supp 1):75-7. doi: https://doi.org/10.1007/ s10549-010-1061-2

11. ASCO Meeting Library [Internet]. Alexandria (VA): American Society of Clinical Oncology. c2019. [cited 2018 Dec 07]. Available from: https://meetinglibrary. asco.org

12. Baena-Cañada JM, Estalella-Mendoza S, GonzálezGuerrero M, et al. Influencia de los factores clínicos y biográficos en la calidad de vida de las mujeres durante la quimioterapia adyuvante por cáncer de mama. Rev Calid Asist. 2011;26(5):299-305. doi: https://doi. org/10.1016/j.cali.2011.04.005

13. Bastani P, Kiadaliri AA. Health-related quality of life after chemotherapy cycle in breast cancer in Iran. Med Oncol. 2011;28(Suppl 1):S70-4. doi: https://doi.org/10.1007/ s12032-010-9714-x

14. Cámara RJA, Schwentner L, Friedl TWP, et al. Quality of life during and after adjuvant anthracycline-taxanebased chemotherapy with or without gemcitabine in high-risk early breast cancer: results of the SUCCESS A trial. Breast Cancer Res Treat. 2019;175(3):627-35. doi: http://dx.doi.org/10.1007/s10549-019-05171-6

15. Hatam N, Ahmadloo N, Ahmad Kia Daliri A, et al. Quality of life and toxicity in breast cancer patients using adjuvant TAC (docetaxel, doxorubicin, cyclophosphamide), in comparison with FAC (doxorubicin, cyclophosphamide, 5-fluorouracil). Arch Gynecol Obstet. 2011;284(1):21520. doi: http://dx.doi.org/10.1007/s00404-010-1609-8

16. Hagiwara Y, Shiroiwa T, Shimozuma K, et al. Impact of adverse events on health utility and health-related quality of life in patients receiving first-line chemotherapy for metastatic breast cancer: results from the SELECT BC study. Pharmacoeconomics. 2018;36(2):215-23. doi: http://dx.doi.org/10.1007/s40273-017-0580-7

17. Au HJ, Eiermann W, Robert NJ, et al. Health-related quality of life with adjuvant docetaxel- and trastuzumabbased regimens in patients with node-positive and high-risk node-negative, HER2-positive early breast cancer: results from the BCIRG 006 study. Oncologist. 2013;18(7):812-8. doi: http://dx.doi.org/10.1634/ theoncologist.2013-0091

18. Browall M, Ahlberg K, Karlsson P, et al. Health-related quality of life during adjuvant treatment for breast cancer among postmenopausal women. Eur J Oncol Nurs. 2008;12(3):180-9. doi: https://doi.org/10.1016/j. ejon.2008.01.005

19. Gaton-Johansson F, Watkins CC, Kanu IK, et al. The effects of symptoms on quality of life during chemotherapy in African-American women with breast cancer. J Natl Black Nurses Assoc. 2015;26(2):7-16.
20. Gozzo TO, Moysés AMB, Silva PR, et al. Náuseas, vômitos e qualidade de vida de mulheres com câncer de mama em tratamento quimioterápico. Rev Gaúcha Enferm. 2013;34(3):110-6. doi: http://dx.doi.org/10.1590/ S1983-14472013000300014

21. Hall E, Cameron D, Waters R, et al. Comparison of patient reported quality of life and impact of treatment side effects experienced with a taxane-containing regimen and standard anthracycline based chemotherapy for early breast cancer: 6 year results from the UK TACT trial (CRUK/01/001). Eur J Cancer. 2014;50(14):2375-89. doi: https://doi.org/10.1016/j.ejca.2014.06.007

22. Kornblith AB, Lan L, Archer L, et al. Quality of life of older patients with early-stage breast cancer receiving adjuvant chemotherapy: a companion study to cancer and leukemia group B 49907. J Clin Oncol. 2011;29(8):1022-8. doi: https://doi.org/10.1200/ JCO.2010.29.9859

23. Leinert E, Singer S, Janni W, et al. The impact of age on quality of life in breast cancer patients receiving adjuvant chemotherapy: a comparative analysis from the prospective multicenter randomized ADEBAR trial. Clin Breast Cancer. 2017;17(2):100-6. doi: https://doi. org/10.1016/j.clbc.2016.10.008

24. Montazeri A, Vahdaninia M, Harirchi I, et al. Quality of life in patients with breast cancer before and after diagnosis: an eighteen months follow-up study. BMC Cancer. 2008;8:330. doi: https://doi.org/10.1186/14712407-8-330

25. Winters ZE, Haviland J, Balta V, et al. Integration of patient-reported outcome measures with key clinical outcomes after immediate latissimus dorsi breast reconstruction and adjuvant treatment. Br J Surg. 2013;100(2):240-51. doi: : https://doi.org/10.1002/ bjs. 8959

26. Ganz PA, Land SR, Geyer CE, et al. Menstrual history and quality-of-life outcomes in women with nodepositive breast cancer treated with adjuvant therapy on the NSABP B-30 trial. J Clin Oncol. 2011;29(9):1110-6. doi: https://doi.org/10.1200/JCO.2010.29.7689

27. Perroud HA, Alasino CM, Rico MJ, et al. Quality of life in patients with metastatic breast cancer treated with metronomic chemotherapy. Future Oncol. 2016;12(10):1233-42. doi: https://doi.org/10.2217/ fon-2016-0075

28. Shiroiwa T, Fukuda T, Shimozuma K, et al. Comparison of EQ-5D scores among anthracycline-containing regimens followed by taxane and taxane-only regimens for node-positive breast cancer patients after surgery: the N-SAS BC 02 trial. Value Health. 2011;14(5):746-51. doi: https://doi.org/10.1016/j.jval.2011.01.007 
29. Zhang J, Zhou Y, Feng Z, et al. Longitudinal trends in anxiety, depression, and quality of life during different intermittent periods of adjuvant breast cancer chemotherapy. Cancer Nurs. 2018;41(1):62-8. doi: https://doi.org/10.1097/NCC.0000000000000451

30. Ho SSM, So WKW, Leung DYP, et al. Anxiety, depression and quality of life in Chinese women with breast cancer during and after treatment: a comparative evaluation. Eur J Oncol Nurs. 2013;17(6):877-82. doi: https://doi. org/10.1016/j.ejon.2013.04.005

31. Sanford SD, Wagner LI, Beaumont JL, et al. Longitudinal prospective assessment of sleep quality: before, during, and after adjuvant chemotherapy for breast cancer. Support Care Cancer. 2013;21(4):959-67. doi: http:// dx.doi.org/10.1007/s00520-012-1612-7

32. Klemp JR, Myers JS, Fabian CJ, et al. Cognitive functioning and quality of life following chemotherapy in pre- and peri-menopausal women with breast cancer. Support Care Cancer. 2018;26(2):575-83. doi: http:// dx.doi.org/10.1007/s00520-017-3869-3

33. Berger AM, Lockhart K, Agrawal S. Variability of patterns of fatigue and quality of life over time based on different breast cancer adjuvant chemotherapy regimens. Oncol Nurs Forum. 2009;36(5):563-70. doi: https://doi. org/10.1188/09.ONF.563-570

34. Huang SM, Tai CJ, Lin KC, et al. A comparative study of symptoms and quality of life among patients with breast cancer receiving target, chemotherapy, or combined therapy. Cancer Nurs. 2013;36(4):317-25. doi: https:// doi.org/10.1097/NCC.0b013e318268f86d

35. Tachi T, Teramachi $\mathrm{H}$, Tanaka $\mathrm{K}$, et al. The impact of outpatient chemotherapy-related adverse events on the quality of life of breast cancer patients. PLoS One. 2015;10(4):e0124169. doi: https://doi.org/10.1371/ journal.pone.0124169

36. Bernhard J, Zahrieh D, Zhang JJ, et al. Quality of life and quality-adjusted survival (Q-TWiST) in patients receiving dose-intensive or standard dose chemotherapy for high-risk primary breast cancer. Br J Cancer. 2008;98(1):25-33. doi: https://doi.org/10.1038/ sj.bjc. 6604092

37. Montazeri A. Health-related quality of life in breast cancer patients: A bibliographic review of the literature from 1974 to 2007. J Exp Clin Cancer Res. 2008;27(1):32. doi: https://doi.org/10.1186/1756-9966-27-32

38. Muliira RS, Salas AS, O’Brien B. Quality of Life among female cancer survivors in Africa: ah integrative literature review. Asia Pac J Oncol Nurs. 2017;4(1):6-17. doi: https://doi.org/10.4103/2347-5625.199078

39. Ambrósio DCM, Santos MA. Apoio social à mulher mastectomizada: um estudo de revisão. Cien Saude Coletiva. 2015;20(3):851-64. doi: https://doi. org/10.1590/1413-81232015203.13482014
40. Sawada ON, Nicolussi AC, Paula JM, et al. Qualidade de vida de pacientes brasileiros e espanhóis com câncer em tratamento quimioterápico: revisão integrativa da literatura. Rev Latino-Am Enferm. 2016;24:e2688. doi: https://doi.org/10.1590/1518-8345.0564.2688

Recebido em 21.8.2019 Aprovado em 3.2.2020 